\title{
ESCENARIOS PARA LA ACCIÓN COLECTIVA ${ }^{1}$
}

\author{
LUIS MIGUEL MILLER MOYA \\ Instituto de Estudios Sociales de Andalucía (IESA-CSIC). Córdoba
}

\author{
PALABRAS CLAVE ADICIONALES \\ ADDITIONAL KEYWORDS \\ Elección racional, Teoria de juegos, Dilema social, \\ Grupo privilegiado, Masa crítica, Coordinación. \\ Rational Choice, Game Theory, Social Dilemma, \\ Privileged Group, Critical Mass, Coordination.
}

RESUMEN. El presente artículo revisa diversas teorías y modelos sobre acción colectiva. Existen muchos tipos de acción colectiva y que no pueden ser capturados por el mismo modelo formal. Por ello, presentamos tres escenarios a partir de la relación entre los intereses individuales y los colectivos, a saber: (1) dilemas sociales, en los que todos los componentes de un grupo, o la mayor parte del mismo, actuando de acuerdo con sus intereses particulares, obtienen un resultado peor del que hubieran obtenido si hubieran ignorado sus propios intereses; (2) grupos privilegiados, donde un subconjunto de individuos con un nivel de intereses o recursos por encima de la media desempeña un papel crucial en la fase inicial de la acción colectiva, creando, así, las condiciones para la incorporación de otros individuos; y (3) coordinación, donde los actores tienen intereses similares $\mathrm{y}$, aunque puede no importarles qué solución será la impuesta, están de acuerdo en que algún tipo de solución es necesaria.

ABSTRACT. This paper focuses on formal theories and models of collective action. There are many types of collective action, and they cannot all be captured with the same formal model. Taking into account the relationship between individual and collective interests, three types of scenarios for collective action are presented: (1) social dilemmas, in which all or most of the members of a group act following their private interests, obtaining a worse outcome that if they had ignored their own interest; (2) privileged groups, in which a subset of highly interested and/or resourceful people play a crucial role in the early phases of collective action and create the conditions for the incorporation of others; and (3) coordination, in which actors have similar interests, and, although they may not care about which solution is imposed, they all agree that some solution is necessary.

\footnotetext{
${ }^{1}$ Una primera versión de este trabajo fue presentada en septiembre de 2004, en el Grupo de Movimientos Sociales y Acción Colectiva del VIII Congreso Español de Sociología. Agradezco a Fernando Aguiar, Andrés de Francisco, Francisco Linares y Gloria Martínez las críticas realizadas a las distintas versiones del mismo. Agradezco también las sugerencias de modificación de dos evaluadores anónimos de la RIS. Asimismo, este artículo no hubiera sido posible sin las innumerables aportaciones tanto teóricas como metodológicas de Victoria Ateca. Obviamente, la responsabilidad sobre el contenido del texto es enteramente mía.

E-mail: 1mmiller@iesaa.csic.es
}

Revista Internacional de Sociología (RIS)

Tercera Época, No 39, Septiembre-Diciembre, 2004, pp. 167-197. 
RIS

REVISTA INTERNACIONAL DE SOCIOLOGIA

№ 39, SEPTIEMBRE-DICIEMBRE, 2004

LUIS MIGUEL MILLER MOYA

\section{INTRODUCCIÓN}

Abordar el estudio de un problema teórico como el que plantea la acción colectiva no es una empresa sencilla. Muestra de ello es la interminable lista de fenómenos empíricos que se han abordado desde este campo de estudio. No han escapado a la lógica de la acción colectiva desde revoluciones nacionales, concentraciones de protesta, boicots por parte de asociaciones de consumidores o fenómenos de violencia colectiva, hasta el control de la polución o la protección de especies en peligro de extinción; desde la participación en partidos políticos, sindicatos, asociaciones profesionales o asociaciones de vecinos, hasta el cumplimiento con el pago de impuestos o la participación en elecciones democráticas. Pero, dada la heterogeneidad de este conjunto de situaciones sociales, ¿cuál es el elemento común que hace que sean tratadas desde un punto de vista analítico similar?, ¿podemos encontrar diferencias sustanciales entre los distintos ejemplos para afirmar que existe más de una lógica de la acción colectiva? Esta introducción tratará de evidenciar los elementos comunes que comparten aquellas situaciones sociales a las que clasificamos como situaciones o escenarios de acción colectiva. La cuestión de las diferentes lógicas de la acción colectiva será abordada en el resto del trabajo.

Si algo comparten el abanico de situaciones descrito más arriba es que se trata de actividades que requieren de la cooperación o coordinación de dos o más individuos para la consecución de un objetivo común (Sandler, 1992: 1; Oberschall, 1994: 79; Linares, 2002: 82).

La necesidad de cooperación o coordinación sitúa a los individuos ante un entorno estratégico de toma de decisiones (Taylor, 1987: 12; Marwell y Oliver, 1993: 9), es decir, cada individuo, al decidir sobre su participación en la acción colectiva, tiene en cuenta los efectos de la participación de otros individuos. Incluso en situaciones donde los individuos toman decisiones simultáneamente, el actor racional basará su acción considerando la mejor respuesta a las acciones de los demás, debido a que sabe que los demás individuos, actuando racionalmente, harán lo mismo (Coleman, 1990: 30).

De acuerdo con la literatura existente sobre el estudio de la acción colectiva a partir de la teoría de la elección racional, la forma más adecuada de modelar situaciones de acción colectiva donde existe interacción estratégica es a través de los denominados juegos no cooperativos. El uso de este tipo de juegos nos permite pronosticar el resultado derivado estrictamente de la interacción estratégica entre individuos cuyo objetivo es la obtención de un fin común. En un juego no cooperativo debemos tener en cuenta dos elementos, a saber: el conjunto de estrategias del que dispone cada individuo (Morrow, 1994: 66) y el conocimiento común que cada individuo posee acerca de la estructura del juego, así como del resto de jugadores (Gates y Hume, 1997: 9).

Teniendo en cuenta que cada jugador tiene un conocimiento común sobre las 
posibles estrategias, tanto propias como del resto de jugadores, y sobre las reglas del juego, cada jugador intentará elegir la mejor respuesta a las estrategias de los demás. Este hecho es el que nos permite realizar predicciones sobre el comportamiento de actores racionales en situaciones de interacción estratégica. La predicción más importante en un juego no cooperativo es el denominado equilibrio de Nash (EN) formado por un conjunto de estrategias las cuales constituyen la mejor respuesta de cada individuo frente a las estrategias del resto. Alcanzado ese equilibrio ningún individuo tiene incentivos para cambiar de estrategia (Morrow, 1994: 80). Por ejemplo, en una situación de acción colectiva, un individuo decidirá sobre su nivel de contribución para la obtención del bien colectivo teniendo en cuenta los niveles de contribución óptimos (desde un punto de vista individual) de todos los demás individuos (Sandler, 1992: 13). En este sentido, el equilibrio de Nash se constituye en una de las piedras angulares de una aproximación desde la teoría de la elección racional al estudio de la acción colectiva ${ }^{2}$.

Llegados a este punto, nos planteamos el problema clásico de la acción colectiva, a saber: la acción racional individual puede llevar a un resultado socialmente no deseado. Éste es el elemento central del planteamiento olsoniano (Olson, 1971), que ha servido como base para el estudio de la acción colectiva durante las últimas cuatro décadas. Sin embargo, aun manteniendo constante el supuesto de racionalidad contenido en ese modelo, ¿se puede afirmar que todos los problemas de acción colectiva pueden ser reducidos en última instancia a este supuesto de partida?

Algunos de los textos más importantes sobre acción colectiva han concluido, siguiendo el planteamiento de Olson, que el comportamiento racional individual, en situaciones de acción colectiva, conduce a resultados sociales no deseados (Hardin, 1982: 25; Chong, 1991: 5; Taylor, 1987: 19). Este conjunto de aportaciones coinciden en la existencia de una contradicción entre los intereses individuales y el bienestar social ${ }^{3}$. De este modo, los individuos hacen frente a un dilema en el cual tienen que decidir entre seguir sus propios intereses individuales o contribuir al fin común. Pero, volviendo a la pregunta que nos hacíamos más arriba, ¿se trata del único prisma a través del cual analizar las situaciones de acción colectiva? Para responder a esta pregunta, vamos a evaluar los posibles resultados de la acción colectiva en dos niveles, individual y colectivamente. Si con anterioridad hemos mostrado que la forma más refinada de predecir el comportamiento racional individual es el equilibrio de Nash, ahora necesitamos una medida estándar que

\footnotetext{
${ }^{2}$ Aunque, como veremos a lo largo del trabajo, sólo nos aporta una predicción precisa del comportamiento individual cuando el juego que utilizamos para representar la acción colectiva tiene un único equilibrio. En el caso de que existan equilibrios múltiples la utilización del equilibrio de Nash es más problemática.

${ }^{3}$ Para una discusión más amplia sobre el denominado problema de la acción colectiva consultar Aguiar (1991).
} 
nos permita evaluar los resultados de la acción desde un punto de vista colectivo (Sandler, 1992: 13).

Los distintos trabajos que, desde la teoría de la elección racional, han abordado el estudio de la acción colectiva han utilizado el óptimo de Pareto (OP) como estándar para medir la eficiencia del comportamiento colectivo. Este óptimo supone un reparto o asignación de recursos en el cual no es posible mejorar el bienestar de un individuo sin perjudicar al menos el de otro (Sandler, 1992: 13). En otras palabras, "si un resultado es mejor que un segundo para un miembro de la colectividad y al menos tan bueno para todos los demás, entonces la colectividad estará mejor bajo el primer resultado que bajo el segundo. Más aún, todos los resultados que no están dominados por otros constituyen el conjunto de resultados óptimo de Pareto, y la teoría no dice nada acerca de cuál de estos resultados será elegido" (Coleman, 1986, 34) 4

Una vez que decidimos utilizar el equilibrio de Nash y el óptimo de Pareto con el fin de evaluar lo que es racional desde un punto de vista individual y desde un punto de vista colectivo, respectivamente, estamos en disposición de comparar ambas medidas estándar en diferentes situaciones para responder a la pregunta que se encuentra en la base de este trabajo, a saber: $i$ es el escenario dibujado por Olson el único escenario posible para el desarrollo de la acción colectiva?

La comparación entre los intereses individuales y colectivos ha servido a diversos autores (Sandler, 1992; Heckathorn, 1996) para caracterizar distintos escenarios para la acción colectiva. Como se seguirá en este trabajo, la representación de ambos intereses se ha realizado mediante la utilización de juegos no cooperativos en forma estratégica. Sandler (1992: 38 y ss.) distingue fundamentalmente cinco juegos para identificar situaciones de acción colectiva. Éstos son: dilema del prisionero, grupo privilegiado, juego del gallina, batalla de los sexos

\footnotetext{
${ }^{4}$ El criterio de Pareto presenta algunos defectos que merecen ser tenidos en cuenta (Sandler, 1992: 14). En primer lugar, esta medida no nos permite realizar una comparación completa de las preferencias estrictas de cada individuo. En segundo lugar, el criterio de Pareto no dice nada acerca de la distribución de rentas entre los distintos individuos. Se puede cumplir este criterio tanto en situaciones en las que un individuo posee todos los recursos y otro ninguno, como en situaciones en las que se da un reparto equitativo. En tercer lugar, el óptimo de Pareto a menudo favorece el statu $q u o$, debido a que una vez alcanzada esta situación óptima, por ejemplo que alguien tenga todos los recursos y algún otro no tenga nada, se hace imposible mejorar la situación del segundo sin dañar la del primero, lo que iría en contra de la propia definición de este criterio. Como vemos, el criterio de Pareto presenta algunos defectos que plantean serias dudas sobre la conveniencia de su utilización. A pesar de estas debilidades, el óptimo paretiano sigue aportándonos una medida muy valiosa acerca de la eficiencia del comportamiento colectivo, acerca de si un grupo concreto ha logrado maximizar el resultado de una acción teniendo en cuenta los recursos de los que disponía.
} 
y juego de la seguridad ${ }^{5}$. Por su parte, Heckathorn (1996: 258) presenta cinco juegos con el mismo objetivo, a saber: dilema del prisionero, juego del gallina, juego de la seguridad, juego privilegiado y dilema del altruista. El objetivo de este artículo es mostrar cómo este conjunto de situaciones pueden ser clasificadas en tres escenarios, a partir de la utilización de modelos formales (juegos) ya clásicos en el estudio de la acción colectiva, así como de estructuras construidas ex profeso para este trabajo (ver tabla 1).

Tabla 1.

Escenarios para la acción colectiva

\begin{tabular}{llll}
\hline & Escenarios & Relación entre EN y OP & Juegos utilizados \\
\cline { 2 - 4 } & Dilemas sociales & $\mathrm{EN}<\mathrm{OP}$ & Dilema del prisionero \\
\cline { 2 - 4 } $\begin{array}{l}\text { Escenarios } \\
\text { para la acción } \\
\text { colectiva }\end{array}$ & \begin{tabular}{lll} 
Grupos Privilegiados \\
\cline { 2 - 3 }
\end{tabular} & $\mathrm{EN}=\mathrm{OP}$ & Juego de la masa crítica \\
\cline { 2 - 4 } & Coordinación & $\exists$ múltiples EN y/o OP & Juego de coordinación pura \\
& & Juego de la seguridad \\
\hline
\end{tabular}

En primer lugar, consideramos aquella situación en la cual los intereses privados entran en contradicción con los intereses colectivos, en la que todos los componentes de un grupo, o la mayor parte del mismo, actuando de acuerdo con sus intereses particulares, obtienen un resultado peor del que hubieran obtenido si hubieran ignorado sus propios intereses. Este tipo de entornos genera los denominados dilemas sociales (Orbell y Dawes, 1981:38; Van Lange, Liebrand, Messick y Wilke, 1992: 3-4; Komorita, 1994: 8; Kollock, 1998: 183-184), en los que la contradicción entre los intereses privados y sociales da como resultado la no provisión del bien colectivo, o bien la provisión del mismo es inferior a aquella que podría haber obtenido el grupo si hubiera utilizado eficientemente todos los recursos de los que dispone. Debido a esto, no sólo estudiaremos la lógica que rige en los dilemas sociales, sino que discutiremos bajo qué circunstancias puede producirse una superación de los mismos.

El segundo grupo de nuestra tipología reconoce la existencia de un grupo privilegiado. Este término define un grupo en el que algún miembro o subgrupo

\footnotetext{
${ }^{5}$ En realidad en el texto de Sandler podemos encontrar otras matrices, pero éstas son derivaciones de los juegos anteriormente mencionados.
} 
tiene intereses o recursos suficientes para procurar que el bien colectivo sea suministrado, incluso si él mismo tiene que cargar con el coste total de la provisión del bien. Dada esta condición, en presencia de este tipo de grupos se da una coincidencia entre los intereses individuales y los colectivos y, por tanto, no existe dilema social alguno (Heckathorn, 1996: 259).

El tercer caso de nuestro esquema acomoda situaciones en las que el problema central es la coordinación entre individuos. Se trata de situaciones en las que "los actores tienen intereses similares y, aunque puede no importarles qué solución será la impuesta, están de acuerdo en que algún tipo de solución es necesaria" (Wilson y Rhodes, 1997: 767).

\section{DILEMAS SOCIALES}

A lo largo de los años sesenta y setenta del siglo XX varios trabajos académicos pusieron de manifiesto que la conocida afirmación de Adam Smith, según la cual el individuo "persiguiendo su propio interés promueve con frecuencia [el bien] de la sociedad más efectivamente que cuando realmente intenta promoverlo" (Smith, 1937: 423), no era del todo cierta o, al menos, no lo era bajo determinadas circunstancias. Si la afirmación de Smith ha quedado para la posteridad como el germen de la economía de libre mercado, los trabajos de M. Olson (1971 [1965]), G. Hardin (1968) o R. Hardin (1971) significaron la puesta en escena de un importante "fallo de mercado", el denominado "problema de la acción colectiva". Y es que, como pusieron de manifiesto estos autores, la persecución de los intereses puramente individuales, lejos de contribuir al bien común, podría conducir a los individuos al desastre colectivo.

\section{La lógica de la acción colectiva}

Transcurridas cuatro décadas desde su publicación en 1965, la obra de Mancur Olson sigue siendo una referencia obligada para definir el problema de la acción colectiva. La afirmación que se halla en la base del problema olsoniano es que "individuos racionales y auto-interesados no actuarán para alcanzar sus intereses comunes" (Olson, 1965: 2). Pero, ¿en qué se basa Olson para sustentar este argumento ciertamente contraintuitivo?.

El problema de Olson es determinar bajo qué condiciones individuos racionales y auto-interesados contribuirán a la provisión de un bien colectivo y qué nivel de provisión se alcanzará, si es que se alcanza alguno. Para representar la toma de decisión individual Olson utiliza una función de valor, donde queda representado un cálculo simple entre costes y beneficios. En este sentido, se puede afirmar que los individuos funcionan aquí como empresas en un mercado competitivo. Dadas estas condiciones, el autor de The Logic of Collective Action demuestra 
que los sujetos no cuentan con suficientes razones para actuar en pos de un interés común o grupal, a menos que: (a) el tamaño del grupo del que forman parte sea lo suficientemente pequeño o (b) exista coerción o se utilice algún otro tipo de herramienta que incentive dicha acción. De aquí surgen las conocidas aportaciones al estudio de la acción colectiva de este economista norteamericano, a saber: la tendencia individual a no participar en acciones colectivas (problema del gorrón o free-rider) y la necesidad de incentivos selectivos de carácter individual para propiciar la participación de los sujetos en este tipo de acciones ${ }^{6}$.

Algunos años más tarde, la estructura estratégica de la lógica de la acción colectiva o de la tragedia de los comunes fue representada formalmente a través del juego del dilema del prisionero de $n$ personas (Hardin, 1971 y 1982). En su forma más simple, el dilema del prisionero supone dos jugadores interactuando, cada uno de los cuales con dos posibles estrategias, a saber: puede elegir entre la estrategia de cooperar con el otro jugador o la estrategia de defraudar (no cooperar). Debido a que cada uno de los jugadores puede llevar a cabo su estrategia de forma independiente, la combinación de los dos pares de estrategias producen cuatro posibles resultados. La figura 1 muestra los cuatro resultados, representados en cada una de las 4 celdas.

En la figura 1, tanto la fila como la columna bajo el rótulo de "defraudar" tienen los mayores pagos y ésta puede ser considerada como la estrategia dominante $^{7}$ del juego. La estrategia dominante del primer jugador es defraudar, ya que, independientemente de lo que haga el segundo jugador, los pagos que obtiene el primero por defraudar son mayores que los pagos correspondientes a cooperar. Dado que este juego tiene una estructura simétrica, "defraudar" es también la estrategia dominante para el segundo jugador. Al jugar su estrategia dominante, cada jugador defrauda, lo que sitúa a ambos en la celda inferior izquierda de la tabla (P, P). Paradójicamente, el resultado final obtenido es peor que la situación que se hubiera producido si ambos hubieran cooperado (R, R). De este modo, el dilema del prisionero se convierte en un ejemplo paradigmático de un dilema

\footnotetext{
${ }^{6} \mathrm{G}$. Hardin (1968) observa un problema similar en la explotación de los denominados "bienes comunes". Este autor plantea cómo el uso de tierras comunes por parte de pastores puede conducir a una sobreexplotación de las mismas y, por tanto, al desastre colectivo. En este caso, el pastor individual tiene que solucionar el dilema que se le plantea entre aumentar sus cabezas de ganado para obtener un mayor beneficio, a costa de una mayor explotación de los recursos comunes, o no seguir aumentando su producción ganadera, contribuyendo así a la sostenibilidad de los recursos naturales. Este análisis a propósito de las tierras de pasto puede ser trasladado a cuestiones como la sobreexplotación de los bancos de pesca y la polución ambiental, así como a otras problemáticas naturales en las que entran en juego bienes comunes, que suscitan problemas de acción colectiva. Sobre la problemática de los bienes comunes ver Ostrom (1990) y Ostrom et al. (1994).

7 "Una estrategia es dominante si es estrictamente la mejor respuesta a cualquier estrategia que los demás jugadores pudieran elegir” (Gates y Hume, 1997: 35).
} 
RIS

REVISTA INTERNACIONAL DE SOCIOLOGLA

$N^{\circ} 39$, SEPTIEMBRE-DICIEMBRE, 2004

social, donde los individuos, persiguiendo sus intereses privados, obtienen un resultado inferior al que hubieran obtenido si hubieran cooperado entre ellos.

Lo más importante de la aportación de R. Hardin es que vincula la discusión de la lógica que rige en un dilema del prisionero a la lógica de la acción colectiva (Hardin, 1982: 28). Por tanto, dota de una estructura formal a los dilemas sociales que habíamos introducido con anterioridad ${ }^{8}$.

Figura 1.

Matriz genérica de un dilema del prisionero

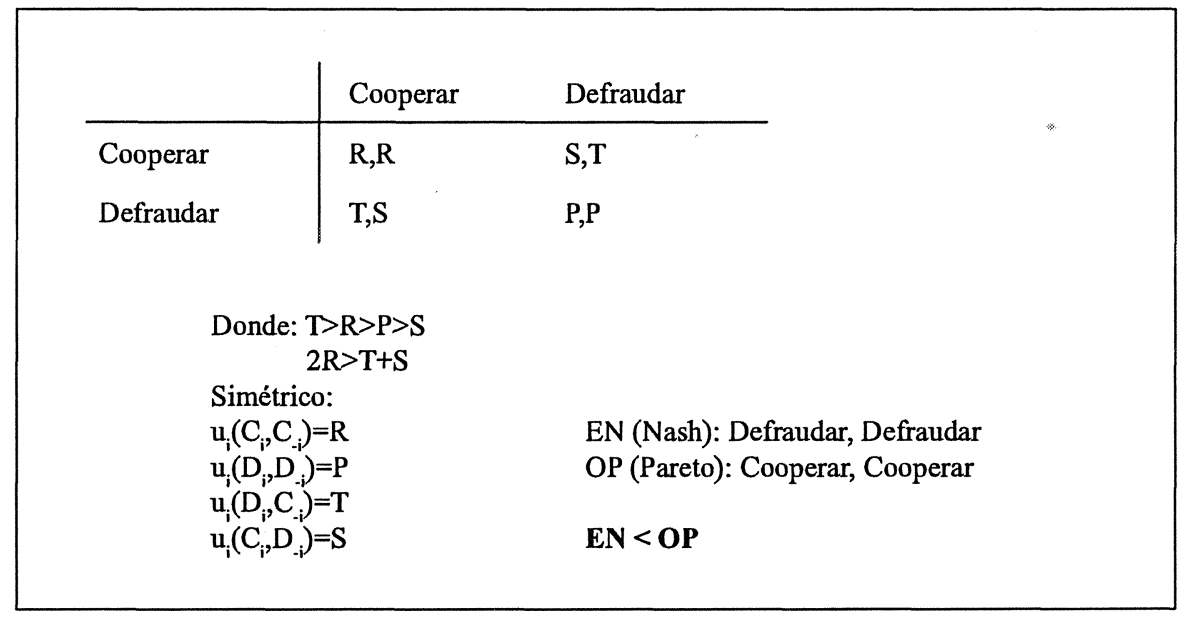

${ }^{8}$ Kollock (1998) realiza un repaso exhaustivo a las distintas formas de representar formalmente un dilema social. La definición de este autor coincide con aspectos ya expuestos en este trabajo, a saber: "los dilemas sociales son situaciones en las que la racionalidad individual conduce a la irracionalidad colectiva" (Kollock, 1998: 183). Más adelante señala que "todos los dilemas sociales están marcados por al menos un equilibrio deficiente" (p. 184) y que "los dilemas sociales más severos están caracterizados por una estrategia dominante que conduce al equilibrio deficiente" (p. 185). La caracterización de un dilema social que hemos realizado en este trabajo se ciñe a cada una de estas características y, aunque asumimos que puede haber dilemas sociales donde no exista estrategia dominante (por ejemplo, en una situación con multiplicidad de equilibrios como puede ser un juego del gallina), hemos preferido tratar el ejemplo más intuitivo de dilema social, el dilema del prisionero, así como otro de los ejemplos más recurrentes: "la tragedia de los comunes". Esta selección de unos juegos y no otros no responde sólo a un intento de claridad expositiva, sino también a las dudas que nos produce caracterizar como un dilema social cada uno de los juegos tradicionalmente utilizados para el estudio de la acción colectiva. Por ejemplo, la utilización del juego de la seguridad para el análisis de diversas situaciones de acción colectiva parece indicar que los individuos insertos en la dinámica propia de este juego no interpretan la situación como un dilema entre los intereses individuales y colectivos, sino como un problema de coordinación (sobre el que abundaremos más adelante). Para un análisis de caso concreto ver Chong (1991). 
Si el comportamiento racional individual, ante un escenario de dilema social, conduce a resultados colectivamente irracionales, ¿cuáles son los mecanismos que permiten la superación de dicho dilema social?

En la literatura especializada en dilemas sociales se han explorado dos tipos de posibles soluciones cooperativas a un problema que adopta la estructura estratégica de un dilema del prisionero, a saber: la cooperación a partir de una situación de dilema del prisionero repetido, y los sistemas de incentivos. A continuación abordaremos de forma resumida la primera, para detenernos posteriormente en la segunda.

\section{Aprender a cooperar}

En un dilema del prisionero repetido es posible por parte de ambos jugadores utilizar amenazas o promesas (implícitas o explícitas) sobre cuáles van a ser los movimientos futuros de ambos jugadores. Esto da origen a un buen número de estrategias e interacciones posibles. Las simulaciones llevadas a cabo por Robert Axelrod (1984) demostraron la efectividad de una estrategia especialmente simple, la denominada estrategia de "toma y daca" (tit for tat), en la cual cada individuo comienza cooperando en la primera ronda y después responde recíprocamente a cada movimiento que el otro jugador haya realizado en la ronda anterior, lo que significa que la cooperación es siempre premiada con cooperación, lo y la defección inmediatamente castigada con defección. Aunque empíricamente puedan darse otro tipo de estrategias por diferentes motivos ("cooperar siempre", "defraudar siempre"), la estrategia de cooperación condicional basada en un toma y daca ha demostrado ser la que muestra una mayor estabilidad al exponerla al paso del tiempo. Además, se trata de un tipo de estrategia que proporciona inequívocas ventajas sociales, tales como que promueve la cooperación entre individuos y no permite que un actor individual sea explotado por el resto.

Sin embargo, esta solución al problema de la acción colectiva se ve condicionada por varias limitaciones que hacen que su aplicación se vea restringida a un conjunto menor de situaciones. En concreto, vamos a explorar tres tipos de limitaciones, de acuerdo con el trabajo de Reuben (2003).

En primer lugar, esta solución descansa sobre un supuesto un tanto irreal por el cual los juegos deben ser repetidos un número infinito de ocasiones, y no parece que esta asunción responda al tipo de situaciones sociales más habituales. Por otro lado, como han mostrado distintos teóricos de juegos, sólo introduciendo la existencia de comportamientos irracionales por parte de determinados jugadores (jugadores que, por ejemplo, siempre mantienen la misma estrategia independientemente de lo que haga el resto) puede conducir a la existencia de cooperación en un dilema del prisionero repetido de forma finita (Kreps et al., 1982).

Un segundo problema de los juegos repetidos es que, para juegos en los que hay más de dos jugadores, las estrategias requieren un gran esfuerzo de coordina- 
ción. Por ejemplo, si un grupo está utilizando una estrategia de toma y daca y un jugador no coopera, el resto de jugadores tendría que cambiar a la estrategia no cooperativa y después volver a la cooperación casi al mismo tiempo. Cualquier jugador que no realice tal cambio de estrategia a tiempo será penalizado de manera injusta. Como el propio Axelrod señala (1997: 41) la simple reciprocidad que puede sostener la cooperación en una interacción repetida entre dos jugadores no es suficiente para el caso de $n$ jugadores, debido a que no permite el surgimiento de las relaciones de confianza o de los sistemas de sanciones que se pueden generar en relaciones de reciprocidad binaria. Además, y esto es lo verdaderamente importante, este tipo de juegos están sujetos a las conocidas conclusiones del "teorema de la tradición oral" (folk theorem). Según éste, cualquier secuencia de acciones cuyo resultado sea mayor que la plena defección mutua puede ser un resultado posible del juego. Por tanto, este modelo no sólo predice que la plena cooperación es un resultado posible, sino que también predice que pueden surgir otros muchos tipos de comportamientos, lo que nos conduce a un resultado muy pobre si lo que buscamos es una teoría con cierto poder predictivo.

Una tercera limitación de la solución al problema de la acción colectiva proporcionada por los juegos repetidos tiene que ver con una de las consecuencias sociales que puede acarrear la interacción repetida a lo largo del tiempo, a saber: el surgimiento de normas sociales e instituciones. El problema es que aún no tenemos un conocimiento adecuado acerca de cómo surgen las normas sociales o de cómo un grupo se provee de una institución que ayude a superar el problema del gorrón (free rider). Y si el surgimiento y mantenimiento de éstas requiere de la presencia de sistemas de incentivos y sanciones nos encontramos ante un problema de acción colectiva de segundo orden, del que daremos buena cuenta en el siguiente apartado.

\section{Incentivos para cooperar}

Descartada la cooperación condicional (a partir de la participación de los actores en una situación de dilema del prisionero repetido) como una solución cooperativa viable a un dilema del prisionero jugado por $n$ personas, en otras palabras, si la simple reciprocidad no es suficiente, la solución propuesta debe pasar por los sistemas de incentivos. $\mathrm{Al}$ menos es la solución que ha proliferado en los trabajos, tanto teóricos como empíricos, que tratan de lidiar con el problema del free rider.

El problema de los sistemas de incentivos y de la "acción colectiva de segundo orden" ha sido ampliamente analizado por autores como Oliver (1980), Coleman (1988 y 1990) y, sobre todo, Heckathorn (1988, 1989 y 1990). En general, se trata de situaciones en las que un agente de control, externo o interno, impone sanciones, bien positivas o bien negativas, sobre un grupo, con el objetivo de incentivar la participación de los individuos en la provisión del bien colectivo. 
Sin pretensión de ser exhaustivos, a continuación vamos a presentar una tipología de incentivos agrupados en torno a tres ejes (ver figura 2). El primero de ellos atiende a la diferenciación realizada por Oliver (1980) entre incentivos positivos (premios) y negativos (sanciones); el segundo aborda la cuestión de si los incentivos son administrados por un agente externo (Heckathorn, 1988) o son puestos en marcha por el propio grupo, así como la relación entre ambos tipos de mecanismos sancionadores (Heckathorn, 1990); en tercer lugar, nos detendremos en la forma concreta que adoptan los incentivos, sean éstos materiales, sociales o morales (Chong, 1991; Opp, 1994).

Figura 2.

Tipos de incentivos selectivos

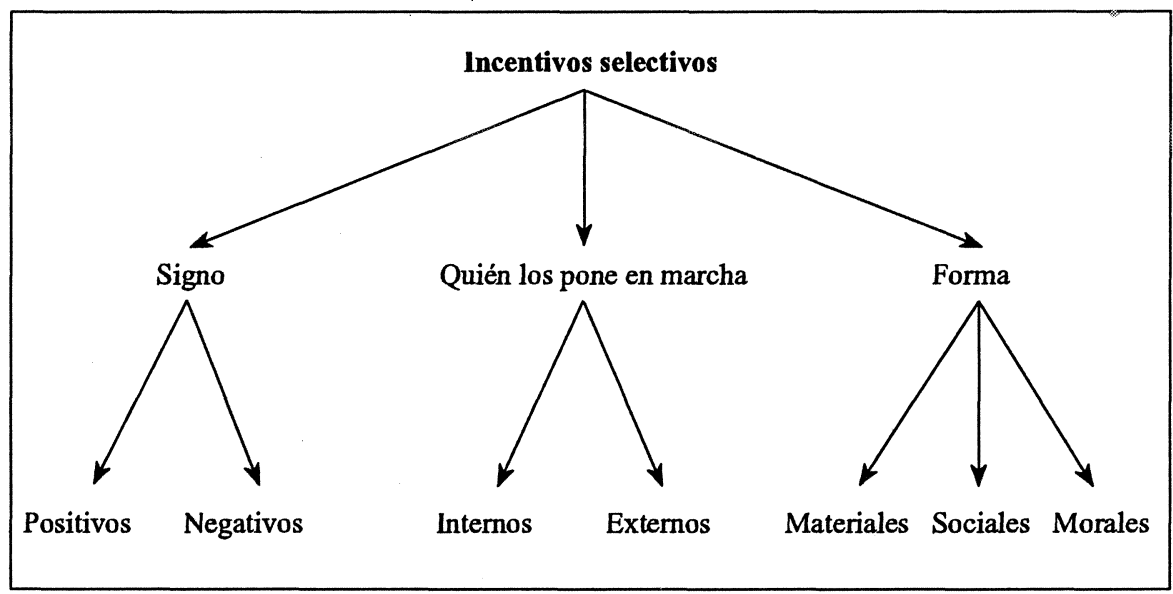

El propio Olson (1971: 51) ya establecía la diferencia entre incentivos selectivos "negativos" y "positivos", los cuales bien pueden actuar como un elemento coercitivo, penalizando a aquéllos que no contribuyan al desarrollo de la acción colectiva, o bien como estímulos ofrecidos a aquéllos que persiguen el interés del grupo. Tomando como punto de partida esta diferenciación, Oliver (1980) argumenta que estos "premios" y "sanciones" suponen mecanismos cualitativamente distintos, cada uno de los cuales funciona con una lógica diferenciada9 ${ }^{9}$. Así, los

\footnotetext{
${ }^{9}$ Esta diferencia no estriba en que afecten de modo distinto a la decisión de cooperar de los individuos, sino que se muestran "radicalmente diferentes desde el punto de vista de la persona que los utiliza" (Oliver, 1980: 1361). Es decir, la diferencia fundamental entre los incentivos selectivos positivos y negativos se halla en el modo en el que son puestos en marcha por aquéllos que administran tales incentivos.
} 
"premios" o "incentivos selectivos positivos", suelen ser más eficaces cuando van dirigidos a un pequeño número de cooperadores, en lo que define como "acciones de élites". Su funcionamiento en grupos grandes queda condicionado a que no exista rivalidad en el consumo de los mismos (es decir, cuanto más se acerquen a un bien público puro en sentido estricto). Ejemplos de incentivos positivos podrían ser las actuaciones de grupos musicales que en numerosas ocasiones acompañan a las grandes manifestaciones políticas o el hecho de repartir entradas de cine entre aquellos escolares que contribuyan a la limpieza del patio del colegio. Por su parte, los "castigos" o "incentivos selectivos negativos" son más eficaces (y económicos) a la hora de estimular la cooperación unánime en grupos que no tienen necesariamente un tamaño pequeño; sin embargo, tampoco están exentos de determinados problemas. Por un lado, se ha demostrado que la eficacia de los mismos es irregular y, en cierto modo, cíclica; por otro lado, su implementación puede generar hostilidades entre los individuos, los cuales pueden adoptar un comportamiento "rebelde" ante las sanciones impuestas. Por último hay que tener en cuenta que en este tipo de sanciones hay que diferenciar dos elementos: la dureza de la sanción y la probabilidad de recibir dicha sanción (por ejemplo, la probabilidad subjetiva de ser víctima de la represión tras participar en una acción de protesta política) (Opp, 1994). En este último caso se trataría de un juego de información imperfecta, donde cada jugador no sabe exactamente la decisión que ha tomado el anterior y tiene que utilizar cálculos de probabilidades subjetivas para tomar la decisión acerca de qué estrategia seguir.

Los trabajos de Heckathorn citados anteriormente se han ocupado principalmente de modelar situaciones en las que un agente de control externo impone sanciones sobre un grupo que persigue un fin colectivo. La cuestión central es que los miembros del grupo en cuestión pueden seguir dos cursos de acción posibles, a saber: pueden cooperar y de este modo no verse expuestos a la sanción, o pueden rebelarse y poner en marcha estrategias dirigidas contra este agente de control externo (Heckathorn, 1988). De este modo, aparece cierto control interno que hace de contrapeso al externo (Heckathorn, 1990), dando lugar a un doble sistema de control, externo e interno. Esta interrelación entre ambos tipos de controles hace desplegar a los actores nuevos tipos de estrategias, como la "cooperación hipócrita" (Heckathorn, 1989; 1993; 1996), o la estrategia desarrollada por aquellos que Coleman (1988; 1990: 273-276) denomina "fanáticos" (zealots). Actuar hipócritamente, en este sentido, supone no contribuir a la provisión del bien colectivo, pero sí participar en la puesta en marcha de sanciones que hagan que el resto coopere. Por su parte, los "fanáticos" son aquellos individuos dentro de un grupo que tienen un exceso de celo respecto de la participación de todos en la obtención del bien colectivo, lo que les hace tener una mayor implicación en la acción colectiva. Algunos ejemplos de individuos o grupos que actúan como "fanáticos" son los voluntarios para estar en la primera línea de fuego en una guerra, o los pequeños grupos que practican un activismo militante (incluyendo 
incluso comportamientos terroristas) en nombre de lo que ellos consideran un "bien público" (Coleman, 1990: 273).

Como ya ha sido puesto de manifiesto en anteriores ocasiones (Linares, 2002: 86), el problema de los sistemas de sanciones es que suponen un "bien colectivo" en sí mismos y, por tanto, darían lugar a un nuevo problema de acción colectiva, la acción colectiva de segundo orden (Coleman, 1990: 270; Oliver, 1993: 286). De esta forma, el argumento plantea algo así como una regresión hasta el infinito, a saber: si para la resolución del problema de acción colectiva necesitamos un mecanismo que supone la emergencia de un nuevo dilema social, siempre existiría algún tipo de dilema no resuelto.

Por último, vamos a establecer una nueva clasificación de incentivos selectivos en función de la forma que adoptan, sean éstos materiales, sociales o morales. Los incentivos selectivos materiales ya fueron ampliamente presentados por el propio Olson, por ejemplo, a través de la discusión que realiza a propósito de las asociaciones médicas. Olson define a estas asociaciones como "gobiernos en miniatura" (Olson, 1971: 138), ya que administran incentivos selectivos como los siguientes: (1) defensa contra las acusaciones de malas prácticas, (2) publicación de revistas médicas que son necesarias para el desarrollo de la profesión, (3) realización de convenciones, no sólo con fines educativos, sino también políticos y sociales. Otro tipo de incentivos materiales pueden ser los fondos que garantizan apoyo económico a los trabajadores que participan en largas huelgas, o la posibilidad de ocupar cargos de relevancia tras un cambio de régimen, como ocurría en las movilizaciones contra los regímenes pro-soviéticos anteriores a la caída del muro de Berlín en la Europa del Este (Opp, 1994: 103).

Los incentivos sociales están relacionados con la presión que el grupo ejerce sobre los individuos. Chong (1991: 35) establece cómo la existencia de fuertes lazos sociales puede ejercer una presión tal que inhiba a los individuos a participar en causas que son consideradas impopulares en su comunidad; por ejemplo, la participación de "blancos" en el movimiento por los derechos civiles de las personas de color en Norteamérica. Pero los lazos sociales también pueden jugar un papel fundamental en el éxito de la acción colectiva, a través de la puesta en marcha de incentivos sociales positivos. Así, está demostrado que la preexistencia de redes sociales desempeña un papel fundamental en la emergencia de la acción colectiva (Chong: 1991,35). En este sentido son muy importantes los trabajos de Taylor $(1976 ; 1987 ; 1988)$ sobre el concepto de comunidad. Un ejemplo histórico puede ser encontrado en los trabajos de Gould $(1991 ; 1993)$ sobre las movilizaciones en la Comuna de París en 1871. Según este autor, las relaciones sociales basadas en redes informales (de vecindad) contribuyeron de forma decisiva al éxito de determinadas movilizaciones y no de otras. Como afirma el propio Gould (1991: 719), "el sistema de reclutamiento residencial, que asignaba las personas a los batallones sobre la base del barrio en el cual vivían, supuso más que un marco organizacional para el esfuerzo insurgente". El hecho de que estos 
RIS

guardias nacionales se hallaran ligados a sus compañeros, no sólo por la causa perseguida, sino también por fuertes lazos sociales, hacía que tuvieran incentivos para "entregarse" más en la batalla y, de este modo, contribuir en mayor medida al éxito de la acción colectiva.

Aunque los incentivos materiales y sociales han sido los que han dado lugar a un mayor número de explicaciones, a la hora de cuestionarnos por las motivaciones para la acción colectiva es necesario tener en cuenta un tercer tipo de incentivos, los incentivos morales. Como afirma Opp (1994: 103), "las personas a menudo sienten que están obligadas a participar cuando se dan ciertas condiciones, como podrían ser determinadas decisiones políticas injustas por parte de las élites políticas". Así, por ejemplo, en las campañas a favor de una mayor igualdad en cuestiones de género, la participación de hombres podría no estar directamente ligada a la expectativa de un incentivo material o social, sino más bien a una cuestión moral relacionada con la persecución de una sociedad más justa e igualitaria.

Hasta aquí han sido expuestas las características propias de un dilema social, así como los medios más recurrentes para superar este problema de acción colectiva. En el siguiente apartado presentaremos el segundo de los escenarios a los que hacíamos referencia en el cuadro 1, a saber: el escenario de los grupos privilegiados.

\section{GRUPOS PRIVILEGIADOS}

La reformulación más importante del problema de la acción colectiva en unos términos distintos al problema del gorrón, es la que a través de diversos trabajos han realizado autores como Marwell y Oliver (1993) ${ }^{10}$. Para Oliver et al. (1985: 546), "el problema de la acción colectiva consiste en conseguir que un conjunto relativamente pequeño de individuos interesados en la provisión de un bien público realicen contribuciones de tiempo, dinero o recursos para la producción de dicho bien".

Este planteamiento nos conduce directamente a la cuestión de los grupos privilegiados, ya planteada por Olson (Olson, 1971: 48-50). Un grupo privilegiado viene definido por el hecho de que algún miembro particular, o un subgrupo de individuos dentro del mismo, tiene incentivos suficientes para procurar que el bien colectivo sea suministrado incluso si él mismo tiene que cargar con el coste total de la provisión del bien. Dada esta condición, en presencia de este tipo de grupos se presupone que el bien colectivo será suministrado y, por tanto, no se

\footnotetext{
${ }^{10}$ En este libro se recogen y reelaboran distintos artículos publicados por ambos autores a lo largo de la década anterior.
} 
presenta el problema de acción colectiva anticipado por Olson. En términos de costes y beneficios, un grupo privilegiado contiene al menos un individuo o una coalición de individuos cuyos beneficios obtenidos a partir de la acción colectiva exceden los costes correspondientes, inclúso si estos costes son soportados únicamente por dicho individuo o coalición (Sandler, 1992: 9). Por tanto, el problema que se presenta en este segundo escenario no está relacionado con la forma más adecuada de superar un determinado dilema (social), sino con las características que debe reunir un grupo para que se trate de un grupo privilegiado.

Como hemos apuntado, la principal característica de un grupo privilegiado es que exista un subgrupo de individuos cuyos mayores intereses o recursos les lleven a realizar una contribución excepcional a la acción colectiva. Los trabajos de Marwell y Oliver (1993), entre otros, han abordado esta cuestión en profundidad. La novedad del planteamiento de estos autores descansa sobre dos supuestos fundamentales, a saber: la cuestión de la heterogeneidad grupal, por una parte, y el estudio de las "funciones de producción" de los bienes colectivos, por otra.

La influencia de la composición del grupo en el éxito de la acción colectiva es una cuestión que ya aparece al comienzo del trabajo de Olson (1971: 3). Éste apunta cómo en determinadas situaciones de acción colectiva se produce una "sorprendente tendencia hacia la explotación de los grandes por los pequeños". En otras palabras, los miembros con mayores recursos e intereses en la obtención del bien colectivo, cargarían con una mayor cantidad de costes y esfuerzos en la obtención del mismo. Este hecho puede resultar discutible; sin embargo, diversos estudios (Sandler, 1992; Marwell y Oliver, 1993; Heckathorn, 1993) han demostrado que la existencia de asimetrías (de recursos, de interés) entre los participantes en la acción colectiva incrementa las posibilidades de éxito de la misma. Pero, ¿bajo qué circunstancias un grupo heterogéneo tiene una mayor probabilidad de conducir con éxito una determinada acción colectiva?

La influencia de la heterogeneidad grupal se ha convertido en uno de los temas más recurrentes para los estudiosos de la acción colectiva. Oliver et al. (1985), a través de una combinación de modelos formales y simulaciones, demuestran que la diferencia de intereses entre los miembros de un grupo favorece siempre el éxito de la acción colectiva, mientras que una desigual distribución de recursos sólo tiene efectos positivos sobre la acción colectiva cuando el bien colectivo que se pretende alcanzar tiene una función de producción creciente ${ }^{11}$. Heckathorn (1993) aporta algunos otros elementos que permiten comprender mejor la influencia de la heterogeneidad grupal en el éxito de la acción colectiva. Para éste, la disparidad de intereses puede tener efectos positivos sobre la acción, ya que "incrementa el número de actores que están motivados para contribuir voluntariamente debido

\footnotetext{
" La cuestión de las funciones de producción de los bienes colectivos será abordada más adelante en este artículo.
} 
R IS

REVISTA INTERNaCIONAL DE SOCIOLOGIA

№ 39, SEPTIEMBRE-DICIEMBRE, 2004

LUIS MIGUEL MILLER MOYA

al alto valor que tiene el bien colectivo (para éstos)" (Heckathorn, 1993: 343). Sin embargo, la existencia de esta "masa crítica" de individuos también puede "bloquear" la incorporación de nuevos participantes, debido a que si los primeros se valen por sí mismos para la obtención del bien colectivo, los segundos no encontrarán motivaciones suficientes para emprender la acción. Heckathorn (1993: 346) también concluye que la heterogeneidad de recursos tiene un efecto positivo sobre la acción colectiva.

Como adelantábamos anteriormente, para Oliver et al. (1985), el efecto que puede tener la heterogeneidad grupal sobre la acción colectiva guarda una estrecha relación con el tipo de función de producción del bien colectivo del que se trate. A continuación nos detendremos en esta relación y cómo bajo determinadas circunstancias podemos encontrarnos ante un grupo privilegiado.

\section{Funciones de producción y masa crítica}

La cuestión que a partir de Oliver et al. (1985) ha cobrado una mayor relevancia en este sentido es que, en general, las acciones colectivas se enfrentan a un problema de puesta en marcha (start up problem), debido a que nadie tendría incentivos a priori para iniciar la acción. Este problema ya fue planteado en cierto modo por Granovetter (1978), dando origen a otra de las líneas de investigación más fértiles sobre acción colectiva: los modelos de umbral. Sin embargo, Oliver et al. (1985) plantearon que este problema en la fase inicial de la acción colectiva podía ser superado a través de lo que, tomando el concepto desarrollado por Schelling (1978: 89), denominaron "masa crítica". La masa crítica es definida por estos autores como "un subconjunto de petsonas especialmente interesadas o con una cantidad importante de recursos que desempeñan un papel crucial en las primeras fases de la acción colectiva" (Oliver y Marwell, 2001: 296). En este sentido, la existencia de una masa crítica de individuos contribuiría a que el grupo latente (utilizando la terminología de Olson) se constituyera en un grupo privilegiado, resolviendo así el problema de la acción colectiva.

Para entender cómo la "teoría de la masa crítica" aporta una solución diferente al problema de la acción colectiva, debemos tener en cuenta dos elementos, a saber: cómo toman los individuos la decisión de participar en acciones colectivas y cómo son agregadas tales decisiones. La primera de estas cuestiones fue formulada por Oliver (1980) a través de la siguiente ecuación (la cual representa el cálculo que realiza cada individuo a la hora de decidir si participa o no en una acción colectiva, así como cuánto aportará a la misma):

$$
\mathrm{G}_{\mathrm{i}}(\mathrm{r})=\mathrm{v}_{\mathrm{i}}[\mathrm{P}(\mathrm{R}+\mathrm{r})-\mathrm{P}(\mathrm{r})]+\mathrm{I}-\mathrm{C}_{\mathrm{i}}(\mathrm{r}),
$$

donde $G_{i}$ representa la ganancia neta para cada individuo; $C_{i}$ el coste individual de contribución al bien colectivo; $r$ la contribución individual al bien colectivo; 
$R$ la contribución del resto de individuos al bien colectivo; $P$ la cantidad de bien colectivo que es alcanzado basado en la contribución total $(R+r$ si $i$ contribuye, y $R$ si $i$ no contribuye); $v_{i}$ supone el valor de $P$ para cada individuo; por último, $I$ es el valor de cualquier incentivo selectivo de carácter privado para un determinado individuo. A través de un simple cálculo, podemos predecir que existirá cooperación si el beneficio neto individual es mayor que cero $\left(G_{i}(r)>0\right)$. Una pequeña manipulación de la ecuación de decisión (1) nos permite afirmar que ello ocurre cuando:

$$
[\mathrm{P}(\mathrm{R}+\mathrm{r})-\mathrm{P}(\mathrm{r})]>\left(\mathrm{C}_{\mathrm{j}}(\mathrm{r})-\mathrm{I}\right) / \mathrm{v}_{\mathrm{i}}
$$

El término $P(R+r)-P(r)$ es una función de producción, la cual nos ofrece la diferencia en el beneficio $P$ producida por una contribución $r$. Este algoritmo matemático ha sido utilizado como parte de modelos más complejos de toma de decisiones en contextos de interdependencia estratégica, en los que pueden hallarse individuos que difieren tanto en el grado de interés que muestran en la obtención del bien colectivo, como en el nivel de recursos que poseen. Oliver et al. (1985) asumen que los individuos toman decisiones de forma secuencial, y muestran cómo los grupos heterogéneos se comportan de forma diferente dependiendo de la forma de la función de producción. Es en ese contexto en el que una masa crítica de individuos puede desempeñar un papel crucial en la acción colectiva. En determinados casos, la función de producción tiene rendimientos marginales decrecientes, por lo que la diferencia $P(R+r)-P(r)$ se hace más pequeña a medida que el número total de contribuciones se incrementa: en este caso, la masa crítica puede suministrar el bien colectivo mientras que el resto "gorronea". En otros casos, la función de producción tiene rendimientos marginales crecientes, por lo que la diferencia $P(R+r)-P(r)$ se hace mayor a medida que el número total de contribuciones se incrementa: en este caso, la masa crítica posibilita la superación de los costes iniciales de la acción y crea las condiciones necesarias para que el resto participe ${ }^{12}$. A continuación vamos a discutir este último argumento a través de una función de producción en forma de $S$ (ver figura 3$)^{13}$.

\footnotetext{
${ }^{12}$ Algunos ejemplos de bienes colectivos con una función de producción con rendimientos marginales decrecientes son una huelga de hambre o la quema de una bandera. Se trata de acciones que consiguen una gran repercusión en cuanto son puestas en marcha sin que sea necesaria una incorporación masiva de individuos para el éxito de la acción. Por otra parte, las huelgas o los boicots tendrían una función de producción con rendimientos marginales crecientes, ya que, en este tipo de acciones, cuanto mayor sea el número de participantes, mayor es el éxito de la acción.

${ }^{13} \mathrm{La}$ elección de la función de producción con forma de $\mathrm{S}$ no es arbitraria. Autores como Oberschall $(1980,1994)$ o Marwell y Oliver (1993) consideran a este tipo de función como la que
} 
Figura 3.

Función de producción en forma de S con problema de puesta en marcha

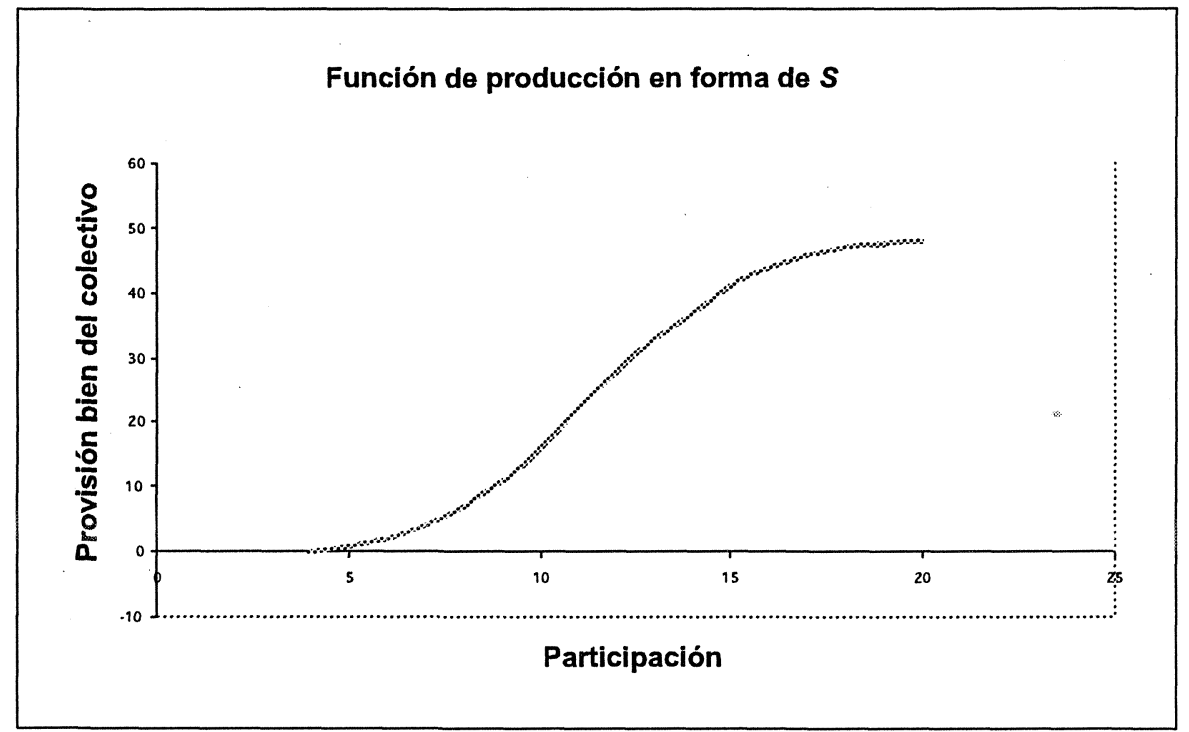

Como se observa en la figura 3, el problema que aquí se plantea es que, hasta que no ha sido alcanzado cierto umbral de participación, no se produce ninguna provisión del bien colectivo; éste es el problema de puesta en marcha (start up problem) propio de las situaciones de acción colectiva que se acomodan a este tipo de funciones de producción. Anteriormente señalábamos que una de las soluciones más recurrentes para este problema es la existencia de una masa crítica de individuos que, en tal caso, estén dispuestos a iniciar la acción incluso en ese primer momento en el que la repercusión es insignificante. Dada este situación, ¿cuál es la estructura de pagos subyacente que hace que este grupo de individuos actúe de forma diferenciada? La respuesta a esta pregunta puede ser encontrada en la figura 4.

mejor acomoda situaciones de acción colectiva como las manifestaciones y protestas colectivas, donde una pequeña acción suele tener poco impacto hasta que se alcanza un determinado nivel de participación; alcanzado éste, nuevas incorporaciones no suponen un mayor impacto de la acción. En la parte central de la función, incorporaciones adicionales incrementan la visibilidad y el impacto de la acción. 
Figura 4.

Juego de la masa crítica

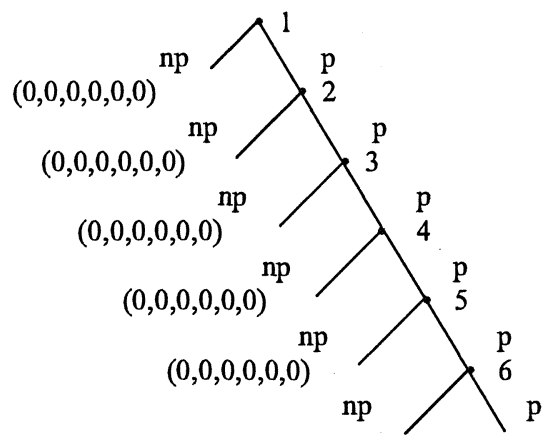

$\left(\Pi_{1} \mathrm{y}, \Pi_{2} \mathrm{y}, \Pi_{3} \mathrm{y}, \Pi_{4} \mathrm{y}, \Pi_{5} \mathrm{y}, 0 \mathrm{y}\right) \quad\left(\Pi_{1} \mathrm{y}, \Pi_{2} \mathrm{y}, \Pi_{3} \mathrm{y}, \Pi_{4} \mathrm{y}, \Pi_{5} \mathrm{y}, \Pi_{6} \mathrm{y}\right)$

Movimientos para ambos jugadores:

$\mathrm{NP}=$ no participar

$\mathrm{P}=$ participar

$\Pi_{1}, \ldots \ldots . . ., \Pi_{6}=$ proporciones en la asignación de bien colectivo alcanzado

$\Pi_{1}+\Pi_{2}+\ldots . .+\Pi_{6}=1$

$\Pi_{1}>\Pi_{2}>\Pi_{3}>\Pi_{4}>\Pi_{5}>\Pi_{6}$

$y=f\left(\sum_{i=1}^{n} \mathrm{c}^{\prime}\right)=$ suma de las contribuciones individuales

$\mathrm{c}=$ contribución individual

El juego de la masa crítica presentado en la figura 4 intenta captar la lógica subyacente a ese primer momento de la acción colectiva donde se produce el problema de inicio. Dicha lógica se asienta sobre dos supuestos fundamentales, a saber: los individuos toman las decisiones de forma secuencial y existen asimetrías (de intereses y recursos) entre los participantes. Formalmente hemos representado la interacción entre seis individuos con distintos intereses en la obtención del bien colectivo. Para ello hemos asignado a cada uno una proporción distinta en el reparto de los beneficios obtenidos de la acción colectiva. Así, al primero que se incorpora a la acción le correspondería una proporción $\Pi$, del beneficio total de la acción $(y)$, al segundo una proporción $\Pi_{2}$, $\mathrm{y}$ así sucesivamente. De este modo, incorporarse pronto a la acción reporta unos mayores beneficios a los participantes que esperar a que se haya incorporado un mayor número de individuos. Este tipo de diseño refleja bastante bien lo que ocurre en multitud de movilizaciones sociales, donde los "pioneros" en emprender.la acción pueden sumar al beneficio que les 
RIS

REVISTA INTERNACIONAL DE SOCIOLOGÍA

NNo 39, SEPTIEMBRE-DICIEMBRE, 2004

LUIS MIGUEL MILLER MOYA

reporta la consecución del bien colectivo, un beneficio privado asociado, ya sea éste social (convertirse en un líder), moral (ser el primero en levantarse contra una situación injusta), o material (obtener un cargo o posición preeminente tras la movilización). Para el individuo $l$ el cual asumimos que conoce perfectamente la forma de la función de producción a la que aludíamos en la figura 4, así como su nivel de interés, encontrará racional participar en la acción, adelantando que su participación contribuirá de forma definitiva al comienzo de la acción. En nuestro ejemplo (función de producción en forma de $S$ y heterogeneidad de intereses), los cuatro primeros individuos encuentran racional participar en la acción, ya que adelantan una situación futura en la que una cantidad mayor de individuos va a participar, generando un mayor nivel de producción del bien colectivo y unos mayores beneficios para los que han puesto la acción en marcha. Si volvemos a nuestra figura 3, a partir del quinto individuo la función de producción es creciente y, por tanto, es completamente racional la participación de nuevos individuos, aunque éstos no tengan un interés en la obtención del bien colectivo tan elevado como los "pioneros" o "masa crítica". Por tanto, nos encontramos ante una situación en la que la heterogeneidad de intereses, unida a la forma que adopta la función de producción del bien colectivo, consigue generar una dinámica de participación masiva, al menos hasta que la gráfica alcanza su máximo y comienza a decrecer. Esta situación, además de suponer una situación de equilibrio desde el punto de vista de la racionalidad individual (equilibrio de Nash), también es una situación óptima desde un punto de vista colectivo (óptimo de Pareto).

En resumen, en este apartado hemos comprobado que, asumiendo que existe disparidad de intereses y recursos entre los participantes en una acción colectiva, en determinadas situaciones (grupos privilegiados), los intereses individuales coinciden con los colectivos y, por tanto, no se reproduce la lógica olsoniana propia de los dilemas sociales.

\section{COORDINACIÓN}

Si la lógica de los dilemas sociales estaba caracterizada por un contraposición entre intereses individuales y colectivos, y la lógica subyacente a los grupos privilegiados partía de situar la heterogeneidad de intereses y recursos en el punto de partida de la explicación, en este tercer escenario no es necesario introducir ninguno de estos dos supuestos. En general, los denominados "problemas de coordinación" han servido para denominar situaciones en las que "los actores tienen intereses similares y, aunque puede no importarles qué solución será la impuesta, están de acuerdo en que algún tipo de solución es necesaria" (Wilson y Rhodes, 1997: 767).

Cuando pensamos en "problemas de coordinación" enseguida nos vienen a la mente situaciones tan cotidianas como por qué lado de la carretera circular, en qué 
lugar he quedado con un compañero para un almuerzo de trabajo, o quién vuelve a llamar cuando se produce un corte inesperado en una llamada telefónica ${ }^{14}$. Sin embargo, no sólo estos problemas simples requieren de soluciones coordinadas, sino también cuestiones como, por ejemplo, la formación de una muchedumbre de manifestantes, la segregación racial entre individuos de un mismo barrio (Schelling, 1960: 90-91), la participación en manifestaciones políticas u otras medidas de protesta (Chong, 1991; Oberschall, 1994), o la identificación con grupos étnicos o nacionales (Hardin, 1995).

Si bien no se han prodigado los estudios sobre cómo opera la coordinación en situaciones sociales reales, la literatura que ha analizado este tipo de lógicas desde un punto de vista formal, así como la utilización de experimentos de laboratorio para su estudio, se ha desarrollado bastante a lo largo de las últimas décadas ${ }^{15}$. Dos de los primeros investigadores que anticiparon la importancia de los problemas de coordinación fueron Schelling (1960), en su influyente libro The Strategic of Conflic, y Lewis (1969), el cual utilizó los presupuestos formales desarrollados por el propio Schelling para modelar las "convenciones sociales" como un juego de coordinación.

Desde un punto de vista formal, el problema que se da en el contexto estratégico propio de los juegos de coordinación es que existen múltiples equilibrios de Nash, es decir, no existe una predicción clara sobre cuál será el resultado del juego. Como muestra el juego de la figura 5 , ninguno de los jugadores tiene una preferencia estricta por una estrategia concreta que se encuentre en su campo de posibilidad; sin embargo, ambos prefieren coincidir con el otro jugador en la estrategia escogida. Además, en este juego coincidir en la estrategia elegida resulta ser una situación óptima desde un punto de vista colectivo, mientras que la puesta en práctica de estrategias diferentes conduciría a un resultado subóptimo del juego. Pensemos, para ilustrar este juego, en el ejemplo clásico de la decisión acerca de por qué lado de la carretera conducir. En una situación ideal de partida, un conductor sería indiferente sobre por qué lado conducir, siempre que todos los demás conductores lo hicieran en el mismo sentido ${ }^{16}$.

\footnotetext{
${ }^{14}$ Otros ejemplos recurrentes pueden ser encontrados en el influyente trabajo de Lewis (1969: 5-8).

${ }^{15}$ Un repaso actualizado de las cuestiones formales que plantean los problemas de coordinación, así como de su puesta a prueba en experimentos de laboratorio, puede ser encontrado en Ochs (1995) y Camerer (2003).

${ }^{16}$ Como es bien sabido, este problema de acción colectiva ha sido resuelto por la evolución de distintas convenciones en diferentes paises; mientras que en zonas como en la Europa continental o Estados Unidos la convención es circular por la derecha, en Gran Bretaña o Australia se circula por la izquierda.
} 


\section{RIS}

Figura 5.

Juego de coordinación pura

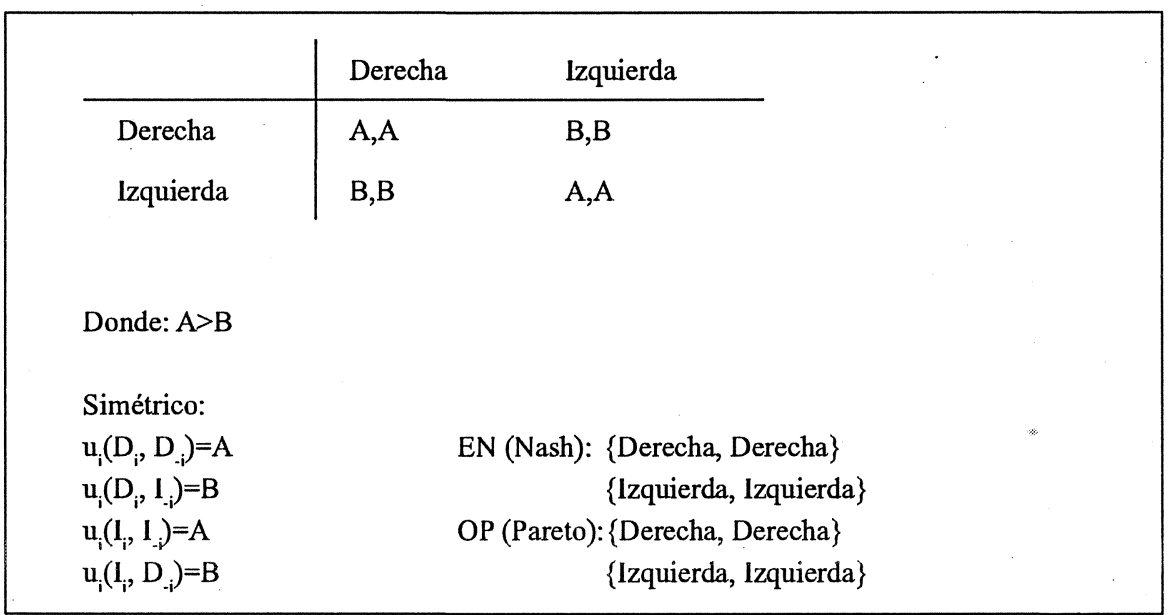

Para la teoría de juegos, la cuestión de cómo coordinan los individuos satisfactoriamente sus acciones es un misterio (Janssen, 2001). Si existen dos o más equilibrios de Nash, la teoría no es capaz de explicar por qué los individuos acaban coordinándose en uno de estos equilibrios y no en otro ${ }^{17}$. Buena parte de los manuales (ver, por ejemplo, Rasmusen, 1989, o Kreps, 1990), se refieren al hecho de que los jugadores, para coordinarse, utilizan puntos focales (Schelling, 1960) o convenciones sociales (Lewis, 1969). Sin embargo, ambas nociones plantean serios problemas a la teoría de juegos más estándar. A partir de estos trabajos, existe una abundante literatura que ha explorado desde un punto de vista teórico dos soluciones recurrentes a los problemas de coordinación, a saber: la existencia de "puntos focales" y la utilización de "señales" que guíen la propia coordinación. Entre los estudios que han explorado la primera, destacan aquéllos que consideran el "liderazgo" como uno de los elementos críticos a la hora de resolver problemas de coordinación (Frohlich et al., 1971; Hardin, 1982; Chong, 1991; Wilson y Rhodes, 1997).

La cuestión de las "señales" está estrechamente relacionada con la existencia de puntos focales en torno a los cuales alcanzar soluciones coordinadas. Así, como demuestran Wilson y Rodees (1997), la eficacia de un líder para conseguir

\footnotetext{
${ }^{17}$ Aunque se exploren vías como analizar qué equilibrio aporta unos mayores beneficios, o cuál es el menos arriesgado, la cuestión de la selección de equilibrios es un tema completamente abierto en la literatura actual sobre teoría de juegos.
} 
la coordinación de un grupo dependerá de lo más o menos creíbles que resulten las señales que éste emite. Esto da paso a otra serie de elementos que pueden ser decisivos para el estudio de la coordinación en situaciones de acción colectiva, tales como la reputación y las relaciones de confianza. Un último elemento que no debe olvidarse en este breve repaso sobre la literatura más reciente sobre "problemas de coordinación" es la cuestión del riesgo (Sally, 2002), es decir, cómo los individuos acaban coordinándose en situaciones que, si bien no son las que aportan un mayor beneficio, son las que comportan un menor riesgo.

Como ejemplo, pensemos en una situación de protesta laboral. Parece claro que la participación de todos los trabajadores supondría un beneficio mayor para el conjunto; sin embargo, un buen número de este tipo de medidas de protesta acaba fracasando por la negativa de los trabajadores a participar. Podríamos recurrir a la explicación de la presencia o ausencia de incentivos selectivos para participar, pero, como han mostrado autores como Chong (1991, capítulo 6) y Oberschall (1994: 84 y ss.), la dinámica que se produce en situaciones de protesta puede ser interpretada de forma más clara mediante el juego de la seguridad y la cuestión del riesgo. En una protesta todo el mundo sabe que sólo una alta participación garantiza el éxito de la misma ${ }^{18}$. Además, no existe una especial tendencia al free riding debido a que si se vislumbra que la acción va a ser exitosa los individuos querrán participar en ella. Por tanto, el problema individual que cada uno tiene que resolver es hasta qué punto el resto de trabajadores está comprometido con la acción, puesto que sólo participará si lo hace un número importante de individuos.

La coordinación, en este sentido, se podría resolver a través de la comunicación entre los participantes, la cual puede contribuir a disminuir la incertidumbre acerca de cómo se van a desencadenar los hechos. Como señalábamos anteriormente, otros elementos que pueden facilitar la coordinación son la puesta en escena de líderes que indiquen hacia dónde tiene que ir la acción ${ }^{19}$, o la existencia de símbolos, señales y otros puntos focales que logren captar la atención del grupo ${ }^{20}$.

Hasta ahora hemos introducido de forma intuitiva diferentes argumentos acerca de cómo opera la coordinación en situaciones de acción colectiva. Para

\footnotetext{
${ }^{18}$ Klanderman (1988: 90) utiliza un argumento parecido para analizar la participación en sindicatos. En su análisis comprueba que si la mayoría de los miembros creen que sólo pocas personas participarán en una determinada acción, puede ocurrir que este hecho desencadene una espiral de deserciones entre los miembros del propio sindicato. Al contrario, si el sistema logra generar participación, es muy posible que la misma sirva como refuerzo para llamamientos futuros. Por tanto, nos encontramos ante un problema básico de coordinación.

${ }^{19}$ Baste con recordar los ejemplos de Luther King o Gandhi como símbolos en torno a los cuales se coordinaron sendos movimientos de protesta con unas características muy definidas.

${ }^{20}$ En este caso, el papel desempeñado por los medios de comunicación es muy importante. Este es el caso, por ejemplo, de cómo la presencia mediática del Movimiento Antiglobalización ha
} 
RIS

REVISTA INTERNaCionaL DE SOCIOLOGIA

No 39, SEPTIEMBRE-DICIEMBRE, 2004

LUIS MIGUEL MILLER MOYA

concluir este apartado, vamos a intentar mostrar formalmente dos cuestiones, a saber: ¿bajo qué condiciones puede producirse un cambio de escenario de la lógica de la cooperación a la lógica de la coordinación? y ¿qué importancia tiene la información en la resolución de los problemas de coordinación?

\section{¿Cómo nos coordinamos? Una interpretación a partir del juego de la seguridad}

El juego de coordinación que ha servido para captar un mayor número de situaciones sociales es el denominado juego de la seguridad. Podemos encontrar intentos iniciales de captar la interacción social que se produce en este tipo de estructura en los trabajos de Schelling (1960), Sen (1967; 1974) o Elster (1979). Ya en los años noventa, la representación del componente de interacción social presente en cualquier movilización colectiva a través de un juego de la seguridad fue tratado por autores como Chong (1991), Oberschall (1994) o Cortazar (1997). La interacción que se produce en una situación representada por un juego de la seguridad responde a los juegos presentados en las figuras 6 y 7 .

Para situar el juego presentado en la figura 6, supongamos que dos líderes vecinales (A y B) deben decidir si apoyan una protesta contra el gobierno local. El problema es que el líder B no conoce el tipo del otro líder, teniendo, de este modo, que afrontar una situación en la que tiene información incompleta ${ }^{21}$. Por tanto, se enfrenta a dos escenarios distintos dependiendo del tipo de líder con el que intente negociar. El primer escenario (tiene enfrente un líder del tipo I) le conduce hacia una situación propia del juego de la seguridad, es decir, la opción más valorada es la cooperación mutua y no existe tendencia a intentar al free-riding. Sin embargo, si el jugador A es un líder del tipo II, el escenario cambia sustancialmente, nos encontramos en una situación propia del dilema del prisionero, donde la opción preferida es que el otro participe en la protesta, mientras que yo no me implico y obtengo una doble ventaja: por una parte, me beneficio de los resultados positivos que se pueden derivar de la propuesta $\mathrm{y}$, por otra, no me comprometo frente al gobierno local (que está en contra de la movilización) y me hallo en mejores condiciones para negociar con el mismo de forma unilateral.

posibilitado su consolidación como movimiento global (Jiménez y Alcalde, 2002). En este sentido, tanto la prensa escrita, como, sobre todo, la televisión, han contribuido sobremanera a la superación de los enormes problemas de coordinación derivados de articular un movimiento de protesta que se ha prodigado desde Seattle a Praga, desde Québec a Barcelona o a Génova.

${ }^{21}$ En un juego de información incompleta, como el mostrado en la figura 6, un movimiento inicial de la "naturaleza" $(\mathrm{N})$ determina con qué probabilidad un jugador es de un tipo u otro. 
Figura 6.

De la cooperación a la coordinación

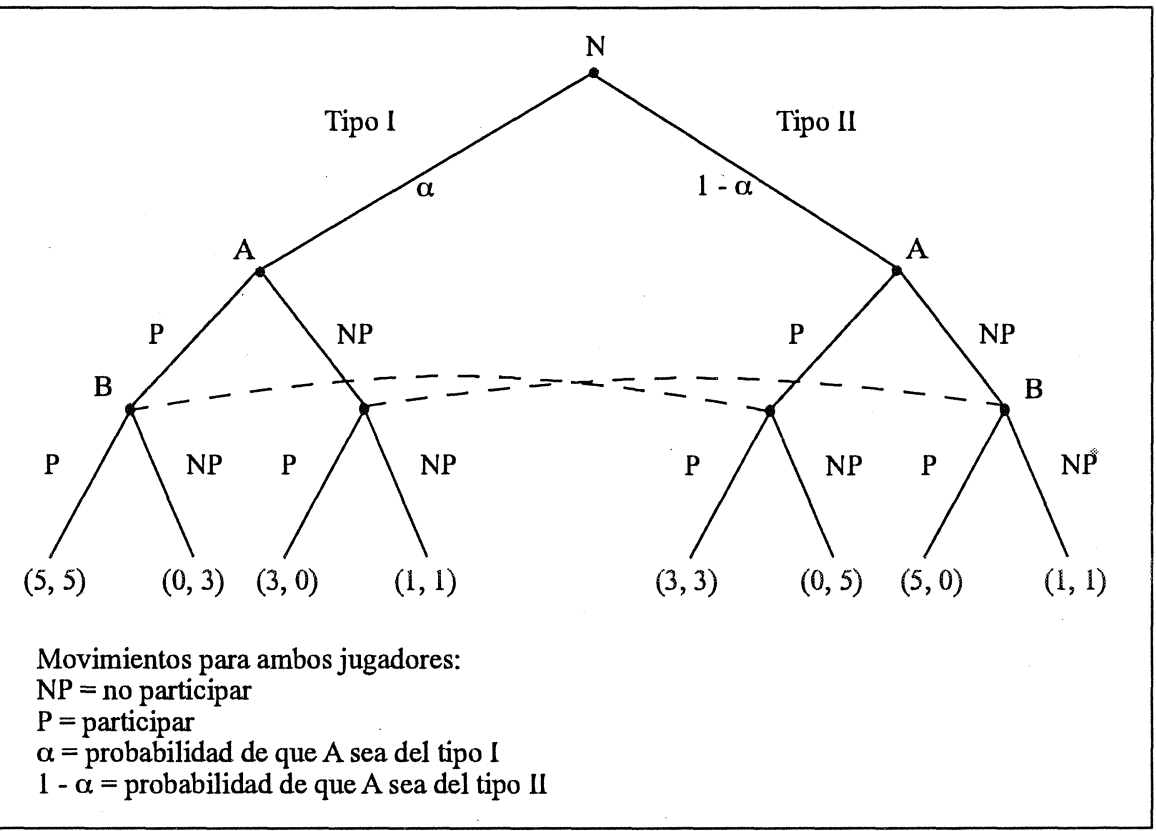

Dada esta situación, lo importante para el jugador B es determinar ante qué tipo de líder se halla para, de este modo, actuar en consecuencia. Así, el juego tiene dos equilibrios dependiendo de las creencias del jugador $\mathrm{B}$ acerca del tipo del jugador $A$. En este sentido, si $\alpha<1 / 3$, es decir, si la probabilidad asignada por $B$ a que A sea del tipo I es menor a un tercio, la estrategia de B será no participar, debido a que este último interpreta que se halla ante una situación asimilable a un dilema del prisionero (la estrategia dominante de A en este caso sería no participar). Por el contrario, si $\alpha>1 / 3$, es decir, si la probabilidad asignada por B a que A sea del tipo I es mayor que un tercio, la situación deriva en un escenario propio del juego de la seguridad (donde la participación mutua es el escenario más valorado por ambos). Como se puede observar, el resultado del juego dependerá de la probabilidad subjetiva que $\mathrm{B}$ asigne a que A sea de un tipo u otro. Este hecho tiene una gran importancia, ya que sólo en el supuesto de que B tenga razones suficientes para creer que $A$ es del tipo I, ambos pueden beneficiarse de la dinámica positiva que se genera al resolver este problema de coordinación, es decir, la participación mutua como resultado más probable. De modo contrario, ambos tendrán que afrontar un dilema social en el que, persiguiendo sus propios intereses, acabarán no participando en la acción colectiva. 
RIS

REVISTA INTERNACIONAL DE SOCIOLOGIA

No 39, SEPTIEMBRE-DICIEMBRE, 2004

En este escenario, A podría intentar influir en las creencias de B para mostrarle a éste que es del tipo I y, por tanto, incitarle a que participe. Se trataría entonces de un juego de señales ${ }^{22}$, y aquí es donde aparece una de las soluciones típicas de un problema de coordinación. A podría lanzar determinadas señales acerca de su voluntad férrea de participar en la acción, lo que cambiaría, sin duda, las creencias de B. Pero las creencias de B, acerca del tipo de A, no sólo pueden verse condicionadas por señales puntuales de este segundo. Otra posibilidad es que B posea información acerca del comportamiento pasado de A, por ejemplo, de su actitud ante convocatorias de protesta anteriores $y$, en función de esta información, actualice sus creencias y modifique la probabilidad subjetiva asignada a que A sea de un tipo u otro. En definitiva, B se encuentra ante una situación de incertidumbre que sólo podrá resolver actualizando sus creencias a partir de la información disponible en el juego ${ }^{23}$.

Hasta aquí hemos visto a través de qué mecanismos un jugador puede encontrarse ante un cambio de escenario, de un dilema social a un juego de coordinación. Pero, como ya avanzamos anteriormente, una vez que asume que se encuentra inserto en un juego de coordinación surgen nuevas situaciones de incertidumbre, como muestra la figura 7.

En la figura 7, el jugador A decide si participa o no en la acción (por ejemplo, la convocatoria de protesta a la que nos hemos referido con anterioridad) y, a continuación, el jugador B decide sobre su participación, aunque, en este caso, sin conocer cuál ha sido el movimiento del primero. Se trata de una situación de información imperfecta que capta perfectamente la lógica de coordinación presente en un juego de la seguridad. Dada la estructura de pagos de este juego, el resultado predicho es que ambos jugadores se coordinarán dos terceras partes de las ocasiones en el equilibrio que implica la participación de ambos (siendo éste el único óptimo de Pareto del juego) y una tercera parte de las veces se coordinarán para no participar.

\footnotetext{
${ }^{22}$ En un juego de señales, el jugador $\mathrm{A}$, a veces llamado emisor, mantiene cierta información oculta que afecta a la decisión que va a tomar el jugador B, el cual es llamado receptor. El primer jugador tiene la oportunidad de emitir dicha información de forma previa a que el segundo jugador tome su decisión (Morrow, 1994: 222). Hay que tener en cuenta que la utilización de señales por parte del jugador A representa un coste para éste. Por ejemplo, si el líder A pretende dar una imagen de líder activo, comprometido con la comunidad, digno de confianza, etc., tendrá necesariamente que invertir tiempo y esfuerzo en lograr que la señal que emite sea creíble. El tamaño de este coste de señalización influirá en la utilización que el jugador A haga de las señales.

${ }^{23}$ Un interesante ejemplo histórico sobre las dificultades de coordinación entre distintos líderes puede encontrarse en el tratamiento que realiza Herreros (2003) a propósito del apoyo de los partidos socialdemócratas alemán y francés a la participación de esos países en la Primera Guerra Mundial.
} 
Figura 7.

Juego de la seguridad

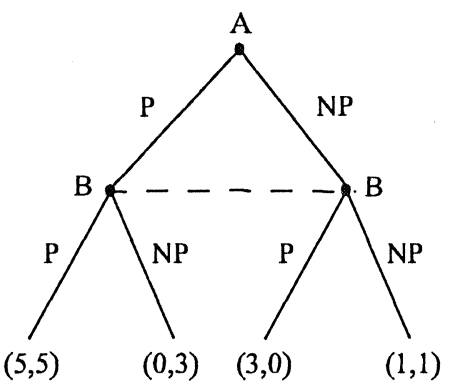

De nuevo, el resultado teórico del juego puede ser modificado al introducir nuevos elementos en el mismo. Al igual que en la situación anterior, el resultado puede ser modificado si ambos jugadores son de distinto tipo, si utilizan señales o si la cuestión del riesgo desempeña un papel central.

El juego de la seguridad, así como otros ejemplos de "problemas de coordinación", sólo son la "punta del iceberg" de la que puede convertirse en una prometedora línea de investigación futura en el terreno de la acción colectiva. $\mathrm{Su}$ desarrollo dependerá de la capacidad para trasladar los avances realizados en modelos formales y situaciones de laboratorio a problemas empíricos concretos.

\section{CONCLUSIONES}

El objetivo central de este trabajo ha sido presentar los distintos escenarios ante los que nos podemos encontrar en una situación de acción colectiva. Son numerosos los enfoques y las herramientas que podríamos haber utilizado para emprender este trabajo. Sin embargo, hemos preferido sacrificar parte de la riqueza que plantea un análisis tan complejo como éste, pretendiendo aportar una tipología de escenarios lo suficientemente simple para que pueda servir de base a investigaciones futuras. En aras de tal claridad y simplicidad, nuestra apuesta ha sido comprobar teóricamente el abanico de posibilidades que se deriva de la contraposición de los intereses individuales con los intereses colectivos. ¿Por qué creemos que esta propuesta de análisis es la más adecuada para nuestro objetivo principal? La respuesta a esta pregunta nos lleva directamente al comienzo de nuestra reflexión: la obra de Olson.

El escenario social que se deriva de la lógica olsoniana plantea una contradicción expresa entre los intereses individuales y el bienestar del colectivo. Éste es 
un hecho fundamental para entender una buena parte de los problemas de acción colectiva, pero no es el único. Para demostrar la existencia de otros escenarios posibles, nos hemos planteado las distintas combinaciones que se pueden dar al contraponer la racionalidad individual y la colectiva.

La tipología presentada al comienzo de este trabajo muestra tres escenarios para la acción colectiva que, desde un punto de vista formal, son diferentes entre sí. El primero, al que hemos denominado dilema social, corresponde a una desigualdad entre el equilibrio de Nash (racionalidad individual) y el óptimo de Pareto (bienestar colectivo). En el segundo caso, en el escenario de la masa crítica, ambas medidas coinciden, por lo que no se produce dilema alguno. En tercer lugar, planteamos situaciones con un denominador común: multiplicidad de equilibrios; es decir, no tenemos una predicción unívoca sobre cuál va a ser el resultado del juego que intenta captar el problema de acción colectiva.

Pues bien, si aceptamos que la clasificación presentada en la introducción nos facilita una base para modelar formalmente situaciones de acción colectiva, o una determinada forma de acercarnos a cuestiones empíricas, podemos estar en condiciones de construir sobre estos escenarios simples explicaciones mucho más complejas. Éste era el resultado esperado del presente trabajo; una vez definido el terreno en el que nos movemos, resultará más sencillo hacernos preguntas en profundidad sobre las motivaciones que conducen a la acción colectiva, sobre aspectos dinámicos de la misma o sobre qué relación existe entre los distintos tipos de bienes colectivos y los diferentes escenarios propuestos.

\section{REFERENCIAS BIBLIOGRÁFICAS}

AGUIAR, F. (1991), "La lógica de la cooperación”, en F. Aguiar, Intereses individuales y acción colectiva, Madrid, Editorial Pablo lglesias.

AXELROD, R. (1984), The Evolution of Cooperation, Nueva York, Basic Books.

(1997), The Complexity of Cooperation: Agent-based Models of Competition and Collaboration, Princeton, Princeton University Press.

CAMERER, C. F. (2003), “Coordination”, en C. F. Camerer, Behavioural Game Theory, Princeton, Princeton University Press.

CHONG, D. (1991), Collective Action and the Civil Rights Movement, Chicago, The University of Chicago Press.

COLEMAN, J. S. (1986), Individual Interest and Collective Action, Cambridge, Cambridge University Press.

(1988), "Free Riders and Zealots: The Role of Social Networks", Sociological Theory, vol. 6 (1), pp. 52-57. 
(1990), Foundations of Social Theory, Cambridge (MA), Harvard University Press.

CORTAZAR, R. (1997), "Non-Redundant Groups, the Assurance Game and the Origins of Collective Action", Public Choice, vol. 92, pp. 41-53.

ELSTER, J. (1979), Ulysses and the Sirens, Cambridge, Cambridge University Press.

FROHLICH, N., et al. (1971), Political Leadership and Collective Good, Nueva York, Princeton University Press.

GATES, S. y HUMES, D. (1997), Games, Information and Politic, Ann Arbor, The University of Michigan Press.

GOULD, R. V. (1991), "Multiple Networks and Mobilization in the Paris Commune, 1871", American Sociological Review, vol. 56, pp. 716-729.

(1993), "Trade Cohesion, Class Unity, and Urban Insurrection: Artisanal Activism in the Paris Commune", American Journal of Sociology, vol. 98, pp. 721-754.

GRANOVETER, M. (1978), "Threshold Models of Collective Behavior", American Journal of Sociology, vol. 83, pp. 1420-1443.

HARDIN, G. (1968), "The Tragedy of the Commons", Science, vol. 162, pp. 1243-48.

HARDIN, R. (1971), “Collective Action as an Agreeable n-Prisoners' Dilemma”, Behavioural Science, vol. 16-5, pp. 472-481.

(1982), Collective Action, Londres, John Hopkins University Press.

(1995), One For All. The Logic of Group Conflict, Princeton, Princeton University Press.

HECKATHORN, D. (1988), "Collective Sanctions and the Creation of Prisoners-Dilemma Norms", American Journal of Sociology, vol. 94, pp. 535-562.

(1989), "Collective Action and the Second Order Free Rider Problem", Rationality and Society, vol.1, pp. 78-100.

(1990), "Collective Sanctions and Compliance Norms: A Formal Theory of Group-Mediated Social-Control", American Sociological Review, vol. 55, pp. 366-384.

(1993), "Collective Action and Group Heterogeneity: Voluntary Provision Versus Selective Incentives", American Sociological Review, vol. 58, pp. 329-350.

(1996), "The Dynamics and Dilemmas of Collective Action", American Sociological Review, vol. 61 , pp. 250-277.

HERREROS, F. (2003), “ The dilemma of Social Democracy in 1914: Chauvinism or Social Dilemma", Rationality and Society, vol. 15(3), pp. 325-344.

JANSSEN, M. (2001), “On the Principle of Coordination”, Economics \& Philosophy, vol. 17 (2), pp. 221-234. 
RIS

REVISTA INTERNACIONAL DE SOCIOLOGIA

NN 39, SEPTTEMBRE-DICIEMBRE, 2004

LUIS MIGUEL MILLER MOYA

JIMÉNEZ, M. y J. ALCALDE (2002), "La construcción de la identidad pública del Movimiento Antiglobalización en España", Revista Internacional de Sociologia, nº 33, pp. 211-235.

KLANDERMANS, B. (1988), "Union Action and the Free-Rider Dilemma”, en L. Kriesberg y B. Misztal (coord.), Research in Social Movements, Conflict and Change, Greenwich, JAI Press.

KOMORITA, S. S. (1994), "Introduction”, en S. S. Komorita, Social Dilemmas, Madison, Brown and Benchmark.

KOLLOCK, P. (1998), "Social Dilemmas: The Anatomy of Cooperation", Annual Review of Sociology, vol. 24, pp. 183-214.

KREPS, D. (1990), A Course in Microeconomic Theory, Princeton, Princeton University Press.

KREPS, D., et al. (1982), "Rational cooperation in the finitely repeated prisoners' dilemma", Journal of Economic Theory, vol. 27, pp. 245-252.

LINARES, F. (2002), "Elección racional y normas sociales: una vuelta más de tuerca", Revista Internacional de Sociologia, $\mathrm{n}^{\circ} 32, \mathrm{pp} .77-99$.

LEWIS, D. (1969), Convention. A Philosophical Study, Oxford, Basic Blackwell.

MARWELL, G. y P. OLIVER(1993), The Critical Mass in Collective Action: A Micro-Social Theory, Nueva York, Cambridge University Press.

MORROW, J. D. (1994), Game Theory for Political Scientists, Princeton, Princeton University Press.

OBERSCHALL, A. R. (1980), "Loosely Structured Collective Conflicts: A Theory and an Application", en L. Kriesberg (ed.), Research in Social Movements, Conflict and Change, Greenwich, JAI Press.

(1994), “Rational Choice in Collective Protest”, Rationality and Society, vol. 6 (1), pp. 79-100.

OCHS, J. (1995), “Coordination Problems”, en J. H. Kagel y A. E, Roth (Eds.), Handbook of Experimental Economics, Princeton, Princeton University Press.

OLIVER, P. (1980), "Rewards and Punishments as Selective Incentives for Collective Action: Theoretical Investigations", American Journal of Sociology, vol. 85, pp. 1356-1375.

(1993), "Formal Models of Collective Action", Annual Review of Sociology, vol. 19, pp. 271-300.

OLIVER, P. et al. (1985) "A Theory of the Critical Mass. I. Group Heterogeneity, Interdependence and the Production of Public Goods", American Journal of Sociology, vol. 91, pp. 522-556.

OLIVER, P. y G. MARWELL (2001), "Whatever Happened to Critical Mass Theory? A Retrospective and Assessment", Sociological Theory, vol. 19, pp. 292-311.

OLSON, M. (1971 [1965]), The Logic of Collective Action. Publics Goods and the Theory of the Groups, Cambridge (MA), Harvard University Press.

196 
OPP, K. D. (1994), "Repression and Revolutionary Action: East Germany in 1989”, Rationality and Society, vol. 6 (1), pp. 101-138.

ORBELL, J. y R. DAWES (1981), "Social Dilemas”, en G. M. Stephenson y J. M. Davis (eds.), Progress in Applied Social Psychology, Nueva York, Wiley, pp. 37-65.

OSTROM, E. (1990), Governing the Commons: the Evolution of Institutions for Collective Action, Cambridge, Cambridge University Press.

OSTROM, E., et al. (1997), Rules, Games \& Common-Pool Resources, Michigan, The University of Michigan Press.

RASMUSEN, E. (1989), Games and Information, Cambridge, Basic Blackwell.

REUBEN, E. (2003), The Evolution of Theories of Collective Action, Tesis de Máster, Universidad de Amsterdam. http://www.tinbergen.nl/ reuben/colaction.pdf

SALLY, D. (2002), “'What An Ugly Baby!' Risk Dominance, Sympathy, and the Coordination of Meaning", Rationality and Society, vol. 14 (1), pp. 78-1008.

SANDLER, T. (1992), Collective Action, Michigan, The Michigan University Press.

SCHELLING, T. (1960), The Strategy of Conflict, Cambridge (MA), Harvard University Press.

(1978), Micromotives and Macrobehavior, Nueva York, Norton.

SEN, A. (1967), "Isolation, Assurance and the Social Rate of Discount", The Quarterly Journal of Economics, vol. 81 (1), pp. 112-124.

(1974), "Choice, Ordering and Morality", en S. Korner (ed.), Practical Reason, Oxford, Basic Blackwell.

SMITH. A. (1937 [1776]), An Enquiry into the Nature and Causes of the Wealth of Nations, Nueva York, Modern Library.

TAYLOR, M. (1976), Anarchy and Cooperation, Londres, Wiley.

(1987), The Possibility of Cooperation, Cambridge, Cambridge University Press.

(1988), "Rationality and Revolutionary Collective Action", en M. Taylor (ed.), Rationality and Revolution, Cambridge, Cambridge University Press.

VAN LANGE, P., W. LIEBRAND, D. MESSICK y H. WILKE (1992), "Introduction and Literature Review", en W. Liebrand, D. Messick y H. Wilke (eds.), Social Dilemmas. Theoretical Issues and Research Findings, Oxford, Pergamon Press.

WILSON, R.K. y C.M. RHODES (1997), "Leadership and Credibility in N-Person Coordination Games", The Journal of Conflict Resolution, vol. 41 (6), pp. 767-791. 